\title{
THE FATE OF CARBOHYDRATES DURING FERMENTATION OF LOW CALORIE BEER
}

\author{
by \\ NIELS BINDESB $\varnothing L L$ NIELSEN ${ }^{1)}$ and FINN SCHMIDT \\ Quality Services, United Breweries Ltd. \\ Vesterfælledvej 100, DK-1799 Copenhagen V. \\ "Present address: Novo Industri A/S, Novo Allé, DK-2880 Bagsværd
}

Keywords: Low calorie beer, amyloglucosidase, carbohydrate composition, dextrins

\begin{abstract}
The carbohydrate composition during fermentation of low calorie beer, or more correctly low carbohydrate beer, has been examined. The low content of carbohydrates in the final beer is achieved by adding amyloglucosidase to the wort at the beginning of the fermentation. Amyloglucosidase is an exoenzyme capable of degrading non-fermentable dextrins almost quantitatively to glucose, which is then fermented.

A full scale production of a low calorie beer "Kalorius" has been followed. The fermentation took three weeks, and was followed by five weeks of storage. Separation and detection of the carbohydrates were done by high performance liquid chromatography using a commercial carbohydrate column and refractive index detection.

The results show an increased glucose content of approximately $60 \%$ on the first day of fermentation. Furthermore, it is found that amyloglucosidase degrades maltose and maltotriose to glucose. This leads to formation of glucose in such quantities that the yeast probably only metabolizes this single carbohydrate during the whole fermentation.
\end{abstract}

\section{INTRODUCTION}

In recent years there has been a growing market in many countries for low calorie beers (lite or light beers), or more correctly low carbohydrate beers.

The low content of carbohydrates is in most situations due to addition of the enzyme amyloglucosidase (AMG) during fermentation of the beer. AMG is an exoenzyme produced by Aspergillus niger and it is capable of degrading dextrins almost quantitatively to glucose.

The uptake of the fermentable sugars by the yeast in a normal lager fermentation is well established $(3,4,7)$. In a normal fermentation sucrose is rapidly hydrolysed to glucose and fructose which are taken up by the yeast cells during the first few days of fermentation. Then the yeast adapts to maltose and maltotriose uptake and their subsequent fermentation takes place.

In low calorie beer production the addition of AMG affects the relative proportion of the fermentable sugars (glucose, maltose, maltotriose), a situation which has not been described in the literature.

However, the isolation of a yeast strain (Saccharomyces diastaticus) which has the ability to utilize starch and dextrins by virtue of its own production of an extracellular amyloglucosidase has been reported (7). The result of growing this

Abbreviations: $\mathrm{AMG}=$ Amyloglucosidase (Glucoamylase, $\mathrm{EC}$ 3.2.1.3); $\mathrm{DP}=$ Degree of polymerization. 
yeast strain in a solution of freeze-dried beer was a clear accumulation of glucose, but information on the other fermentable sugars was not given. Thus it is of major interest to examine the interaction between yeast, AMG and fermentable sugars, especially because one might fear that a rise in the glucose content caused by AMG activity would postpone the adaptation to maltose and maltotriose to such an extent that all fermentable sugars would not be converted at the time of transfer from fermentation to storage. To investigate this, the composition of carbohydrates during the fermentation of a low carbohydrate beer has been examined.

\section{MATERIALS AND METHODS}

\subsection{Materials}

Two brews (brew 2602 and brew 2609) of the brand "Kalorius" from the Carlsberg Breweries were produced from the following raw materials:

$5050 \mathrm{~kg}$ lager malt $\quad(72 \%)$

$200 \mathrm{~kg}$ caramel malt ( $3 \%$ )

$1800 \mathrm{~kg}$ maize grits $(25 \%)$

The brewing materials were mashed into $250 \mathrm{hl}$ water.

After mashing and wort boiling, including the addition of hops, the worts were sent to the fermenting cellar and cooled in a plate cooler. When the worts were down to $9.5^{\circ} \mathrm{C}$, they were led to the fermenting vessels during addition of 5-8 ppm $\mathrm{O}_{2}$ as sterilized compressed air. Here yeast (Saccharomyces carlsbergensis) and AMG $(10 \mathrm{~g} / \mathrm{hl}$ type $150 \mathrm{~L}$, Novo Industri A/S, Denmark) were added.

The brews were each of approximately $600 \mathrm{hl}$.

In contrast to traditional lager fermentation, "Kalorius" has a three week primary fermentation to allow AMG to hydrolyse unfermentable sugars sufficiently. After fermentation, "Kalorius" was stored for a few weeks before bottling and pasteurisation.

\subsection{Methods}

\subsubsection{Sampling}

Every day around 2 p.m. during the three weeks of primary fermentation, a sample of approximately 0.51 of the fermenting beer was taken from the sampling outlet. The first sample was taken before AMG addition. The last sample was taken after 55 days fermentation and storage. At that time the two brews were mixed.

\subsubsection{Pretreatment of samples}

To remove yeast from samples, these were centrifuged (Mistral $6 \mathrm{~L}$ ) at $2400 \mathrm{rpm}$ and $0{ }^{\circ} \mathrm{C}$ for 15 minutes. After centrifugation, $50 \mathrm{ml}$ was taken out of each sample and frozen. These samples were later used for determination of the content of the different carbohydrates; freezing was necessary to avoid enzyme activity.

The rest of the centrifuged samples were filtered and decarbonated by decantation.

\subsubsection{Determination of alcohol and real extract}

Alcohol and real extract were determined with a ServoChem Automatic Beer Analyzer (SCABA, ServoChem, Sweden). The filtered and decarbonated samples were used for the analysis after calibration and analysis of a standard sample.

The calibration samples used were $0 \%, 3.5 \%$ and $7.0 \%(w / w)$ solutions of ethanol. The standard was a beer with approximately $4 \%$ alcohol $(w / w)$; the alcohol content of this was determined by distillation according to the method described in Analytica-EBC (2).

The SCABA, which operates at $20^{\circ} \mathrm{C}$, splits the sample into two parts; one part is used for measurement of the specific gravity and the other is sent countercurrent with dry compressed air $(14 \mathrm{l} / \mathrm{h})$. The air absorbs a proportional amount of alcohol, which is measured by an alcohol sensor.

From these two measurements a microcomputer calculates the other beer parameters: apparent and real extract $\left(E_{A}\right.$ and $\left.E_{R}\right)$, original extract $(\mathrm{OE})$ and real degree of fermentation (RDF).

\subsubsection{Determination of the carbohydrate composition}

The equipment used was a high performance liquid chromatograph (5000 Liquid Chromatograph, Varian, Calif.); a refractive index detector (RI-3, Varian, Calif.) thermostated to $40^{\circ} \mathrm{C}$ was used for detection. The output from the refrac- 
tive index detector was fed into a data system (CDS 401, Varian, Calif.). The data system was programmed to draw a chromatogram, integrate the different peaks and calculate the percentage content from a standard solution. The standard solution was an external standard containing $1 \%$ glucose and $1 \%$ maltose.

The column used was packed with a cation exchange resin in the silver form (type HPX 42A, Bio-Rad Laboratories, Calif.). The column was thermostated to $85^{\circ} \mathrm{C}$, a temperature where there is no risk of AMG activity.

The flow speed was $0.6 \mathrm{ml} / \mathrm{min}$ and deionized water was used as eluent.

Series of malto-oligosaccharides and isomalto-oligosaccharides prepared by partial acid hydrolysis of respectively amylose (Merck) and dextran (Pharmacia) (6) were used for determination of the retention times for the different carbohydrates. The samples were kindly supplied by Dr. B.S. ENEVOLDSEN, Carlsberg Research Laboratory.

Before injection in a $20 \mu$ loop in the high performance liquid chromatograph, it was necessary to decarbonate the beer sample. To avoid the risk of precipitation of $\mathrm{AgCl}$ in the column, chloride was removed by ion exchange (analytical grade mixed bed resin type AG 501-X8D, Bio-Rad Laboratories, Calif.). $2 \mathrm{~g}$ was added to the $50 \mathrm{ml}$ samples followed by magnetic stirring at room temperature for approximately $20 \mathrm{~min}$. The chloride content was reduced from approximately $30 \mathrm{ppm}$ to $1.5 \mathrm{ppm}$ by this treatment.

It was shown that the ion exchange procedure did not affect the results, since the carbohydrate composition of the beer remained unchanged after addition of an internal standard to a lager beer, ion-exchange procedure and analysis on the chromatograph.

To remove all impurities, the samples were filtered through a Millipore filter (Millex-HA, $0.45 \mu \mathrm{m}$, Millipore, Mass.) and a Sep-Pak $\mathrm{C}_{18}$ cartridge (Waters Associates, Mass.) before injection.

\section{RESULTS}

Because of great similarities between the two brews, only the results from brew 2609 have been presented here.

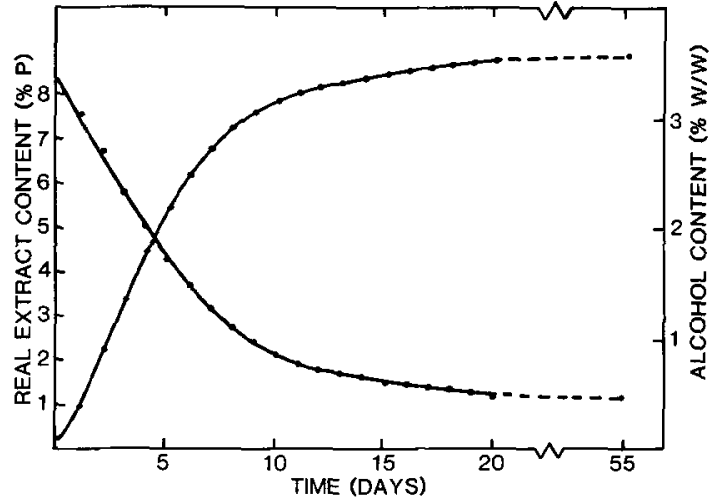

Figure 1. The development in alcohol content (\% w/w ethanol) and real extract content (\% Plato) during fermentation of a low carbohydrate beer. The values are determined by ServoChem Automatic Beer Analyzer.

\subsection{Alcohol and real extract}

The development in alcohol content and the real extract content versus time is shown in Figure 1.

Not surprisingly, the changes are greatest in the first week of fermentation. After the first week the changes become smaller, but it should be noted that there are still changes in the real extract and alcohol content up to the last days before transfer. This fact justifies the relatively long time of fermentation (see section 2.1).

Furthermore, the real degree of fermentation (RDF) can be calculated to $87.4 \%$ after 55 days of fermentation and storage (in conventional lager it is $67-70 \%$ ). This shows the better utilization of the carbohydrates in "Kalorius".

\subsection{Carbohydrate content}

\subsubsection{Qualitative determination}

By means of the series of malto-oligosaccharides and isomalto-oligosaccharides (see section 2.2.4), the retention times of the different carbohydrates in "Kalorius" were determined (Figures 2 and 3 ). The retention times in "Kalorius" were generally a little shorter than the corresponding DP in the above series.

In Figure 2 high amounts of glucose are seen on the fifth day of fermentation whereas in a 


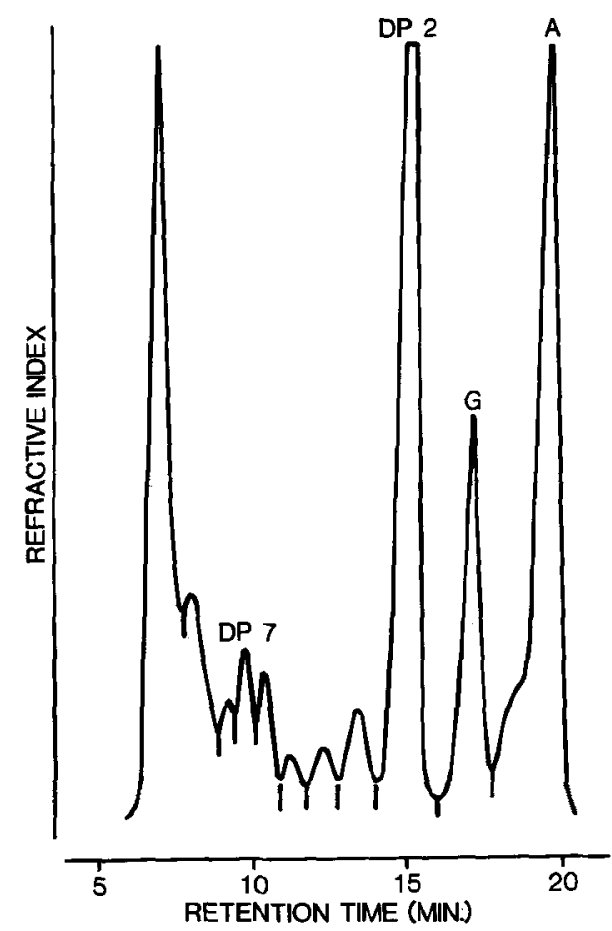

Figure 2. The carbohydrate profile from the fifth day of fermentation of a low carbohydrate beer. The separation is done by high performance liquid chromatography. The peaks indicated are: Alcohol (A), glucose $(G), D P 2$ and DP7. traditional lager fermentation glucose is not present at this time.

After the 17th day (Figure 3) a great deal of the unfermentable sugars has been degraded by $A M G$ and it is seen that the DP2 peak tends to become divided into two with the later top as the greater. The later top is probably isomaltose, which is digested with difficulty by both yeast and AMG. This is supported by the series of malto-oligosaccharides and isomalto-oligosaccharides, where isomaltose has a retention time only 0.2 min higher than maltose.

These results correspond very well with literature (1) which states that AMG has a greater affinity towards the larger oligosaccharides and that AMG cleaves $\alpha(1-4)$ linkages more easily than $\alpha(1-6)$ linkages.

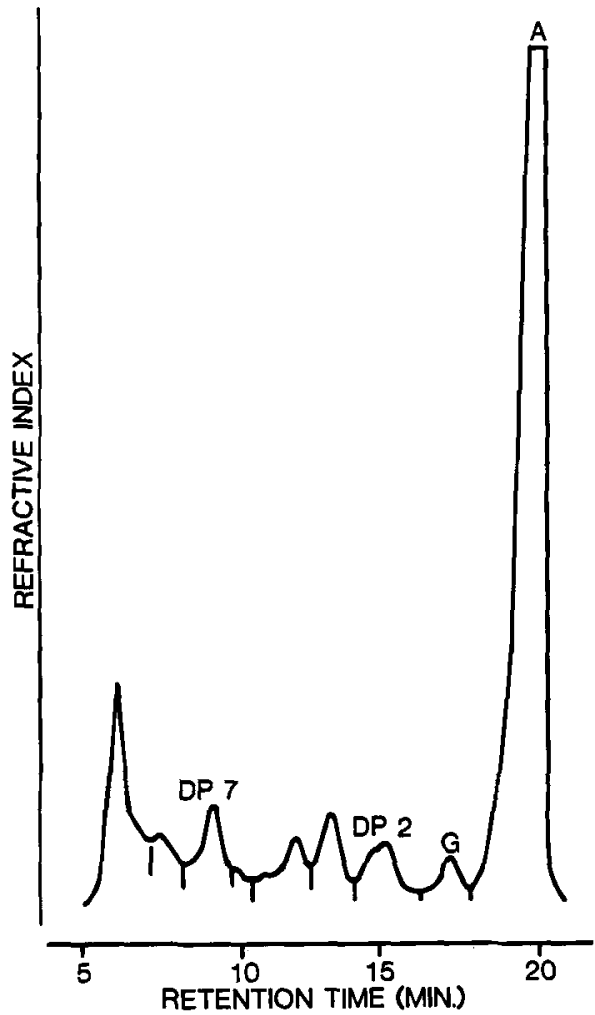

Figure 3. The carbohydrate profile from the 17 th day of fermentation of a low carbohydrate beer. The separation is done by high performance liquid chromatography. The peaks indicated are: Alcohol (A), glucose (G), DP2 and DP7.

\subsubsection{Quantitative determination}

Based on the carbohydrate profiles from days $0,1,2,3,4,5,6,7,10,12,14,17,20,55$, the content of the individual carbohydrates on the different days has been determined as an average of two determinations. The results are shown in the graphs in Figures 4, 5 and 6.

In the beginning there are large amounts of the fermentable sugars, especially maltose (DP2). DP4 is also found, as well as some DP5 and increasing amounts of DP6-8. The content of all components is decreasing in the beginning of the fermentation, with the exception of glucose (Figure 4) where the content is increasing approximately $60 \%$ during the first 24 hours. This content remains constant for the next two days and is then decreasing during the rest of the 
fermentation.

The DP 2 content is decreasing strongly (more than $90 \%$ ) during the first six days of fermentation. In the rest of the period the content moves more slowly towards a constant value, probably isomaltose (see 3.2.1).

DP3 is degraded quite rapidly to a constant value after three days. This value probably represents the content of panose and isopanose which it is difficult for AMG to degrade (1) and not possible for the yeast to metabolize.

DP4-6 (Figure 5) show the following: A decrease followed by an increase and decrease again. The DP5 curve is not very characteristic, yet it seems to follow the above trend.

The picture for DP7-8 (Figure 6) is more complicated, which is due to the fact that the larger the DP is, the larger is the number of isomers involved. The rise in the DP7 content at the end of the fermentation is probably due to formation of double branched heptasaccharides (e.g. $6^{3}$ - $\alpha$-glucosyl- $6^{3}$ - $\alpha$-maltriosyl-maltotriose) which are very difficult for AMG to degrade. DP9 disappears after two days of fermentation, while the DP10-16 peak and the front peak are reduced gradually during the whole fermentation.

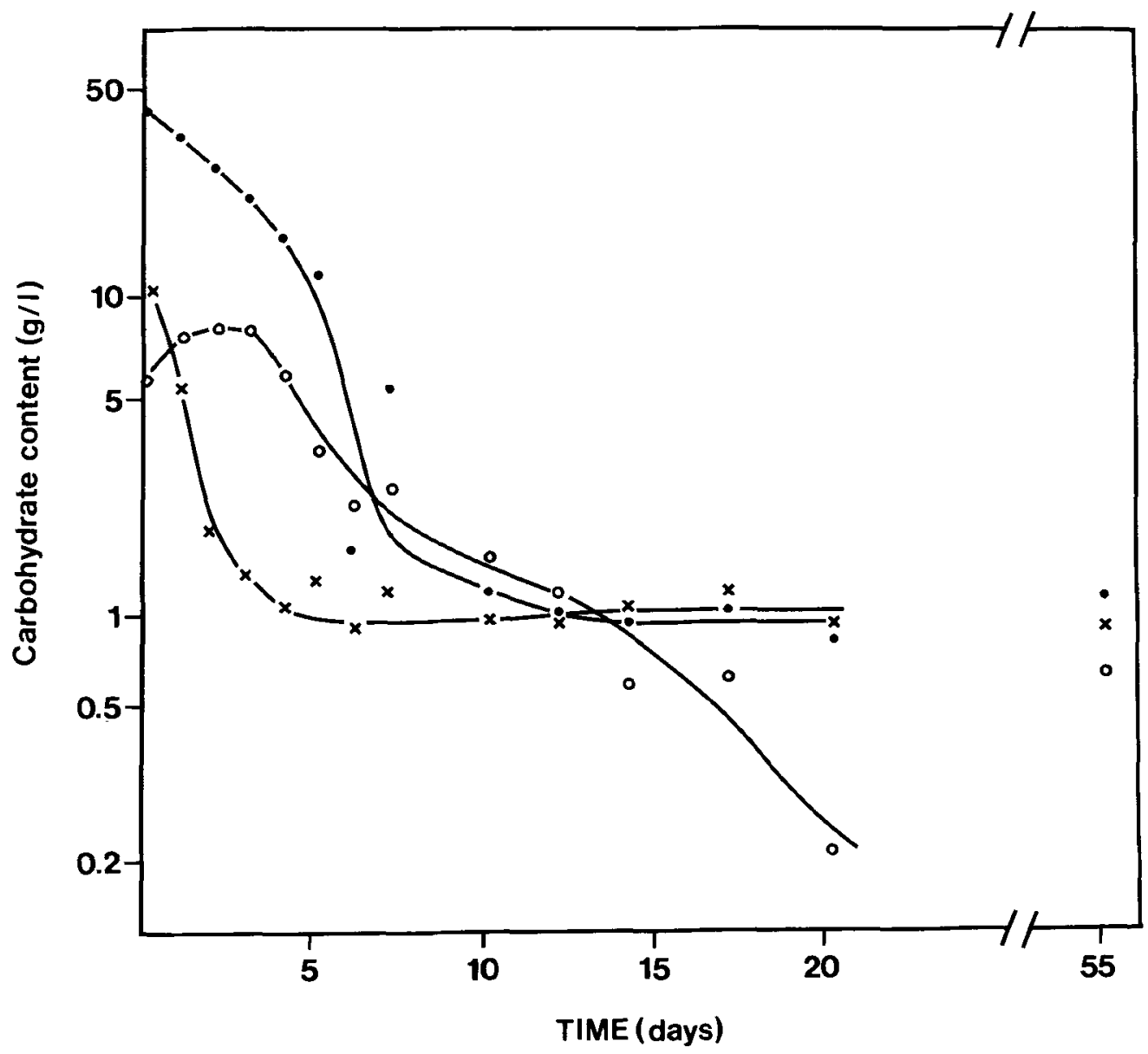

Figure 4. The carbohydrate composition of the fermentable sugars during fermentation of a low carbohydrate beer. Glucose is indicated by ${ }^{\circ}, \mathrm{DP} 2$ by $\bullet$ and DP 3 by $\times$, the values of the components are measured as glucose. 


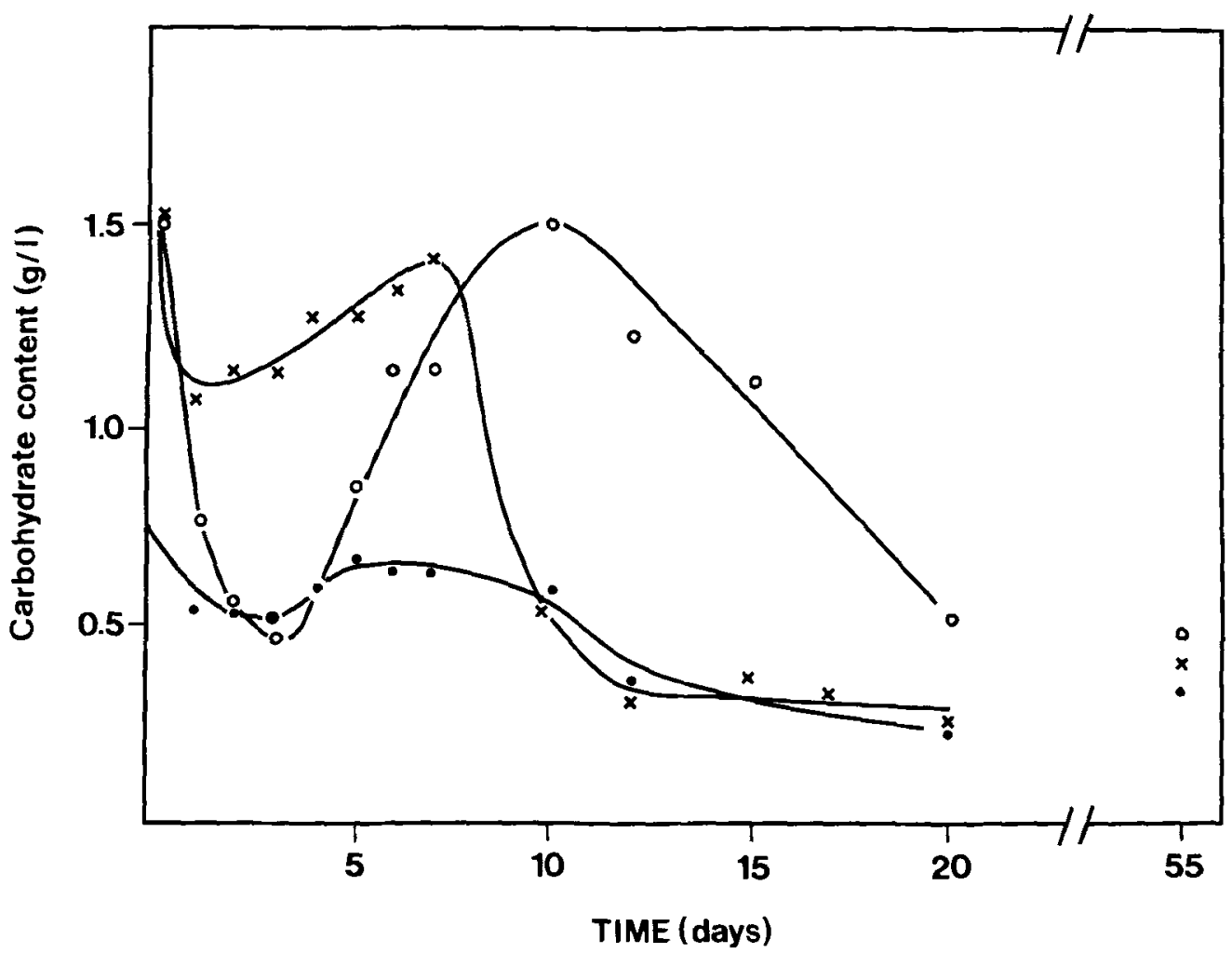

Figure 5. The carbohydrate composition of the smaller dextrins during fermentation of a low carbohydrate beer. DP4 is indicated by 0, DP5 by $\bullet$ and DP6 by $x$, the values of the components are measured as glucose.

\section{DISCUSSION}

In conventional fermentations of beer the amount of dextrins remains unchanged and the alcohol in the beer originates from the fermentable sugars present in the wort before fermentation.

Moreover, in conventional fermentations of beer the fermentable sugars are taken up in a sequential order by the yeast cells, i.e. the monosaccharides, glucose and fructose, disappear prior to the uptake of maltose (which is by far the most dominant of the fermentable sugars) and maltotriose. Thus, in a conventional fermentation the amounts of maltose and maltotriose remain unchanged during the first and second day of fermentation and the onset of the maltose (and maltotriose) fermentation does not take place before the monosaccharides, glucose and fructose, are below a certain low level.

\section{1. $A M G$ and the dextrins}

In contrast to the above conventional fermentation of beer, the addition of amyloglucosidase (AMG) at the beginning of the fermentation leads to an overall reduction in the amount of dextrins and an expected concomitant increase in alcohol, as shown by the results of the present investigation.

In fermentations where amyloglucosidase is present, AMG attacks the individual dextrins thereby liberating glucose and thus increasing the amount of fermentable sugars. The rate at which glucose is liberated from the dextrins depends on the concentration and the susceptibility of the individual dextrins besides the amount of AMG used and the temperature at which the fermentation takes place. When dextrins are degraded to the point where $\alpha(1-6)$ linked glucose units constitute the non-reducing 


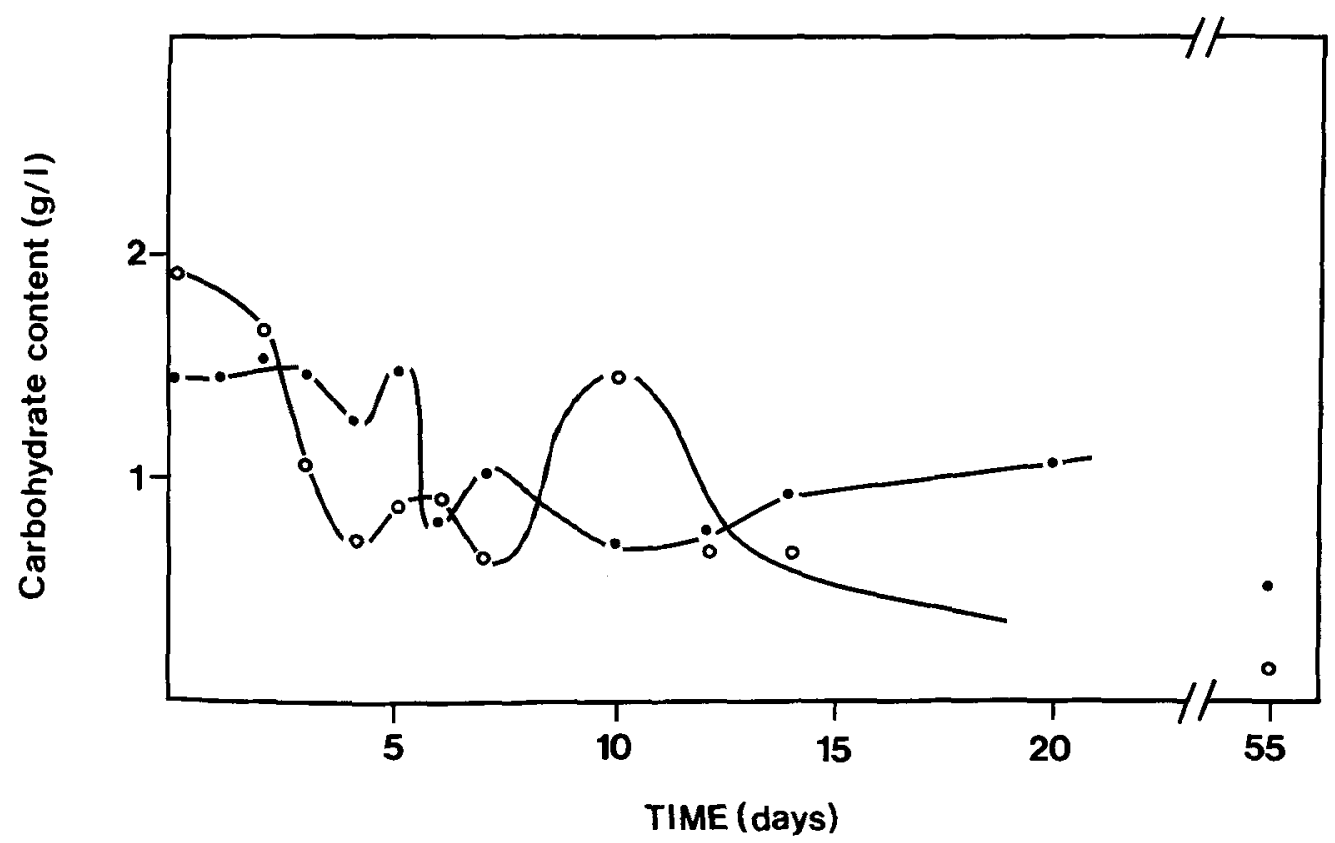

Figure 6. The carbohydrate composition of the larger dextrins during fermentation of a low carbohydrate beer. DP7 is indicated by $\bullet$ and DP8 by $\circ$, the values of the components are measured as glucose.

end(s) of a specific dextrin (oligosaccharide), these dextrins become almost resistant to further attack by AMG at the conditions prevailing for the production of low carbohydrate beers. This means that some dextrins are easily degraded to smaller dextrins (by the stepwise removal of glucose units), which due to their slower rate of hydrolysis may temporarily increase in amount (1), before being further degraded, while some of the dextrins formed, due to their low susceptibility towards amyloglucosidase, tend to accumulate throughout the fermentation period. - The net result, however, is a reduction in the total amount of dextrins and a concomitant formation of glucose, which leads to an increased alcohol content at the expense of the amount of residual carbohydrate (dextrins) conventionally present in beer.

\subsection{AMG and the fermentable sugars}

However, AMG does not only attack the dextrins but is also capable of hydrolysing the fermentable sugars, maltose and maltotriose, to glucose. The question is whether this hydrolysis of maltose and maltotriose actually takes place at the given conditions, and if so, whether it is possible to account for the amounts of maltose and maltotriose that are hydrolysed by AMG to glucose and thus form part of the "pool" of glucose as well as the amounts of maltose and maltotriose that are taken up by the yeast cells as intact oligosaccharides and hydrolysed inside the yeast cell by the yeast maltase ( $\alpha$-glucosidase) to glucose.

The results of the present study are not conclusive in this respect, but the following points, which focus on the interplay of glucose, maltose and maltotriose, are of interest to note.

There is an increase in the amount of glucose (by $60 \%$ ) during the first day of fermentation and this level of glucose is maintained for the following two days (Figure 4). This means that glucose is formed by $A M G$ at a faster rate than the rate at which glucose is taken up by the yeast cells. Furthermore, the results indicate that glucose is 
being produced continuously throughout the fermentation period and for the first 12 days of fermentation is present in an excess of $1 \mathrm{~g}$ per liter.

Thus glucose formation originates from the dextrins as well as from the fermentable sugars, maltose and maltotriose, the levels of which decrease from the very beginning of the fermentation, in contrast to conventional fermentations without $A M G$ addition.

Although it can not be excluded that part of the maltose and maltotriose may be directly fermented, it seems likely that glucose is the only sugar that is fermented in the production of low carbohydrate beers, where AMG is added at the beginning of the fermentation.

If glucose were added continuously from an external source to a conventional fermenting beer, it is anticipated that the presence of glucose would impair the fermentation of maltose and maltotriose and thus lead to sluggish (sticky) fermentations (catabolic repression). This might also be the case in fermentations where glucose is continuously "added" from the dextrins through the action of AMG. However, although the direct fermentation of maltose and maltotriose might be impaired, since the yeast never manages to adapt to the uptake of these oligosaccharides, this does not exclude maltose and maltotriose from being converted to alcohol since the presence of AMG hydrolyses these oligosaccharides outside the yeast cell to glucose, which is then fermented to alcohol.

\section{ACKNOWLEDGEMENTS}

We are most grateful to Dr. B.S. ENEVOLDSEN for stimulating discussions and for critical revisions' of the manuscript. We are endebted to ELSEBETH Sørensen for helping with the manuscript and NINA VIESE for preparing the figures. The technical assistance of BIRGITTE FUNCH JENSEN is gratefully acknowledged.

The investigation is an examination project prepared by N.B.N. for the Department of Technical Biochemistry, The Engineering Academy, Lyngby, Denmark.

\section{REFERENCES}

1. Abdullah, M., I. D. Flemming, P. M. Taylor \& W. J. WHELAN: Substrate specificity of the Amyloglucosidase of Aspergillus niger. Biochem. J. 89, 35P (1963)

2 Analytica-EBC, Analysis Committee of European Brewery Convention, T. -M. Enari ed., Schweizer Brauerei-Rundschau, 3rd ed., Zürich, 7. 1 (1975)

3. ENEVOLDSEN, B. S.: Anvendelse af sukker eller andre kulhydrater til supplering af urtens ekstraktindhold. Beretning om Det XVI Skandinaviske Bryggeritekniske Møde, København, 83-108 (1972)

4. Hough, J. S., D. E. Briggs, R. Stevens \& T. W. YOUNG: Metabolism of wort by yeast. In: Malting and Brewing Science, 2nd ed., Chapman and Hall, London, 566-614 (1982)

5. POMMER, K.: Application of amylolytic enzymes for low calorie beer production. Inst. of Brew. (Aust. \& N. Z. Sect. ), Proc. 17th Conv., Perth, $85-92$ (1982)

6. SCHMidt, F. \& B. S. ENEvoldsen: Gel filtration chromatography of oligosaccharides. Carlsberg Res. Commun. 41, 91-110 (1976)

7. Stewart, G. G., J. Erratt, I. Garrison, T. GorING \& I. HANCOCK: Studies of the utilization of wort carbohydrates by brewer's yeast strain. MBAA Techn. Quart. 16, 1 -7 (1979)

Accepted by S. O. ANDERSEN 